\title{
A CLASSIFICATION OF COHERENT STATE REPRESENTATIONS OF UNIMODULAR LIE GROUPS
}

\author{
WOJCIECH LISIECKI
}

\section{INTRODUCTION}

Let $G$ be a connected Lie group and $(\pi, \mathscr{H})$ a unitary representation of $G$ on a complex Hilbert space $\mathscr{H}$. Throughout we shall assume that $(\pi, \mathscr{H})$ is nontrivial in the sense that $\operatorname{dim} \mathscr{H}>1$. By a coherent state orbit (CS orbit for short) for $(\pi, \mathscr{H})$ we mean a complex orbit of $G$ on the projective space $\mathbf{P}(\mathscr{H})$ (which is equipped with a natural structure of an (infinite-dimensional in general) Kaehler manifold (cf. [L])). We call $(\pi, \mathscr{H})$ a coherent state representation (CS representation for short) if (1) it admits a CS orbit, (2) is irreducible and (3) has (at most) discrete kernel, and we call $G$ a CS group if it possesses CS representations. The purpose of this note is to announce a complete classification of connected unimodular CS groups and their CS representations (Theorems 1 and 2 below). This generalizes the results of EnrightHowe-Wallach [EHW] and Jakobsen [J] on the classification of unitary highest weight (or holomorphic) representations of reductive groups (which coincide with the CS representations as we have shown in [L]). The proofs are "geometric," the main tool being the recent structure theory of homogeneous Kaehler manifolds due to Dorfmeister and Nakajima [DN].

In physics, any orbit on $\mathbf{P}(\mathscr{H})$ is called a system of coherent states in the sense of Perelomov (see [P] and the references therein).

Of particular importance are symplectic coherent state orbits; in many cases such an orbit may be interpreted as the classical phase space of the system whose quantum phase space is $\mathbf{P}(\mathscr{H})$. Such an embedding of the classical phase space into the quantum one is the starting point of Berezin's quantization (see [B1] and [B2];

\footnotetext{
Received by the editors December 1989 and, in revised form, December 11, 1990.

1980 Mathematics Subject Classification (1985 Revision). Primary 22E45, 81D30; Secondary 32M10, 58F05, 58F06.
} 
see also [T] for a comparison of Berezin's quantization with the Kostant-Souriau geometric quantization) and the "quantization of states" proposed recently by Odzijewicz (see [O1] and [O2]). In both theories, the case of complex orbits plays a distinguished role. On one hand, "complex" coherent states are in a sense closest to the classical states [P] and on the other, we may apply in this case powerful techniques of complex analysis (with Bergman type reproducing kernels playing an essential role).

Thus there is a strong physical motivation for studying CS representations.

\section{BASIC PROPERTIES OF CS REPRESENTATIONS}

Here the term CS representation refers to a $(\pi, \mathscr{H})$ which has property (1) but not necessarily (2) and (3).

Proposition 1 [L]. Any CS orbit has a natural structure of a Hamiltonian G-space and the corresponding moment mapping takes it diffeomorphically onto an integral coadjoint orbit with Kaehler (i.e. positive totally complex) polarization.

There is a natural holomorphic line bundle $\mathbf{E}$ over $\mathbf{P}(\mathscr{H})$ whose fiber at $[v]=\mathbf{C} v$ is the dual $[v]^{*}$. The linear dual $\mathscr{H}^{*}$ of $\mathscr{H}$ is naturally isomorphic to the space of holomorphic sections of E. Given a CS orbit $G \cdot[v]$ corresponding to a CS representation $(\pi, \mathscr{H})$, we get a natural map from $\mathscr{H}^{*}$ to the space $\Gamma(G \cdot[v], \mathbf{L})$ of holomorphic sections of $\mathbf{L}$, the restriction of $\mathbf{E}$ to $G \cdot[v]$.

Proposition 2. The following are equivalent.

(i) $v$ is a cyclic vector for $(\pi, \mathscr{H})$.

(ii) The map $\mathscr{H}^{*} \rightarrow \Gamma(G \cdot[v], \mathbf{L})$ is injective.

(iii) $(\pi, \mathscr{H})$ is irreducible.

The implications (i) $\Rightarrow$ (ii) and (iii) $\Rightarrow$ (i) are clear, and (ii) $\Rightarrow$ (iii) can be deduced from a well-known theorem of Kobayashi [K].

\section{THREE SPECIAL CASES}

It turns out that the case of a general unimodular group can be reduced to three special cases, which we shall now briefly discuss.

3.1. Heisenberg groups. Let $H_{n}$ be a $(2 n+1)$-dimensional Heisenberg group (not necessarily simply connected). Identify the (multiplicative) group $\mathrm{X}(C)$ of unitary characters of the center $C$ of $H_{n}$ with an (additive) subgroup of the dual $c^{*}$ of the Lie 
algebra of $C$. The infinite-dimensional irreducible unitary representations of $H_{n}$ are in 1-1 correspondence with the nonzero elements $\lambda$ of $\mathrm{X}(C),\left(\beta_{\lambda}, \mathscr{F}_{\lambda}\right)$ being the unique (up to equivalence) representation with $\lambda$ as central character (or, in other terms, the unique representation corresponding, via Kirillov's bijection, to the integral coadjoint orbit $\mathscr{O}_{\lambda}$ determined by $\lambda$ ). It is well known that any $\left(\beta_{\lambda}, \mathscr{F}_{\lambda}\right)$ is a CS representation. Any of the CS orbits on $\mathbf{P}\left(\mathscr{F}_{\lambda}\right)$ is mapped by its moment onto $\mathscr{O}_{-\lambda}$. This establishes a 1-1 correspondence between these orbits and Kaehler polarizations of $\mathscr{O}_{-\lambda}$ which, in turn, are in 1-1 correspondence with points of the Siegel space $\mathfrak{S}_{n}$ (i.e. the Hermitian symmetric space $\operatorname{Sp}(2 n, \mathbf{R}) / \mathrm{U}(n))$.

Next we consider reductive groups. We shall say that a reductive group is of compact (resp. noncompact) type if its Lie algebra is so.

3.2. Groups of compact type [KS]. Any such group is a CS group and any of its nontrivial representations is a CS representation. For any CS representation, there is exactly one CS orbit, namely the orbit through a highest weight line. Geometrically, these orbits are compact simply connected homogeneous Kaehler manifolds (i.e. flag manifolds).

3.3. Groups of noncompact type [L]. Such a group is a CS group if and only if it is of Hermitian type (i.e. the symmetric space $\mathscr{D}$ associated with it is of Hermitian type). CS representations are the highest weight representations. Again the orbit through a highest weight line is the unique CS orbit for a given CS representation. Geometrically, it is a holomorphic fiber bundle over $\mathscr{D}$ (equipped with one of its invariant complex structures) with flag manifolds as fibers.

\section{Homogeneous KaehleR MaNifolds}

Our approach to the problem of classifying CS groups is based on Dorfmeister-Nakajima theorem [DN] (which gives an affirmative answer to a long standing conjecture of Vinberg and Gindikin). For our purposes, it is convenient to state it as follows. Every homogeneous Kaehler manifold $X$ has a holomorphic double fibration

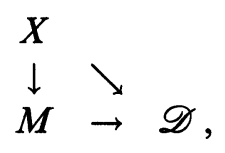

where $M$ is a homogeneous Kaehler manifold without flat homogeneous Kaehler submanifolds and the fibers of $X \rightarrow M$ are flat 
homogeneous Kaehler manifolds (i.e. they are of the form $\mathbf{C}^{n} / \Gamma$, where $\Gamma$ is a discrete subgroup of $\mathbf{C}^{n}$ and the Kaehler metric is induced by the standard Kaehler metric on $\mathbf{C}^{n}$ ), $\mathscr{D}$ is a homogeneous bounded domain and the fibers of $M \rightarrow \mathscr{D}$ are flag manifolds. Such a double fibration is unique and is preserved by all automorphisms of $X$.

\section{STRUCTURE OF A CS ORBIT}

Now suppose $(\pi, \mathscr{H})$ is a CS representation of $G$ and $X=$ $G \cdot[v] \subset \mathrm{P}(\mathscr{H})$ is a CS orbit such that neither its flat fibers nor $\mathscr{D}$ reduce to points. The fact that $X$ is a Hamiltonian $G$-space implies that these flat fibers are isomorphic to some $\mathbf{C}^{n}$ and coincide with the orbits of a Heisenberg group $N$ (of dimension $2 n+1$ ) which is contained in $G$ as a normal subgroup. Let $J_{N}$ denote the moment mapping of the corresponding Hamiltonian action of $N$ on $X$. Since the orbits of this action are symplectic, the symplectic reduction theorem (see [AM]) implies that $J_{N}(X)$ is a single coadjoint orbit $\mathscr{O}_{\lambda}$.

$N$ being a normal subgroup of $G$, there is a homomorphism

$$
\tilde{\rho}: G \rightarrow \operatorname{Aut}(N),\left.g \mapsto \operatorname{Int} g\right|_{N}
$$

(where Int $g$ denotes the inner automorphism of $G$ corresponding to $g$ ), which factors through $N$ to give a homomorphism

$$
\rho: S=G / N \rightarrow \text { Out } N=\text { Aut } N / \text { Int } N \text {. }
$$

It is clear that $\tilde{\rho}(S) \subset(\text { Aut } N)_{\lambda}$, the stabilizer of $\mathscr{O}_{\lambda}$ (or $\lambda$ ) in Aut $N$ (which is the same for all $\lambda \neq 0$ ), and, consequently, $\rho(S) \subset(\text { Aut } N)_{\lambda} / \operatorname{Int} N \cong \operatorname{Sp}(2 n, \mathbf{R})$.

Being a complex submanifold of $X$, each $N$-orbit carries a Kaehler polarization which is mapped by $J_{N}$ into a Kaehler polarization of $\mathscr{O}_{\lambda}$. We thus get a $\rho$-equivariant map from the orbit space $M=X / N$ to the space of all Kaehler polarizations of $\mathscr{O}_{\lambda}$, i.e. the Siegel space $\mathfrak{S}_{n}$. It can be shown that this map is holomorphic. Hence it factors through the compact fibers of $M$ to give a $\rho$-equivariant holomorphic map

$$
\rho_{\mathscr{D}}: \mathscr{D} \rightarrow \mathfrak{S}_{n}
$$

\section{Classification of UNIMOdular CS GROUPS}

From now on we assume that $G$ is unimodular (and nonreductive). Using the results of the preceding section it is not hard to 
show that then $S=G / N$ is also unimodular and so is its quotient $S / N_{\mathscr{D}}$ which acts effectively on $\mathscr{D}$. Moreover, $N_{\mathscr{D}}$ is of compact type (here the assumption that $\pi$ has discrete kernel is essential). Now a theorem of Hano [Ha] asserts that if a unimodular Lie group acts effectively and transitively on a bounded domain, then it is semisimple and the domain is symmetric. Thus $S / N_{\mathscr{D}}$ is semisimple and, consequently, $S$ is reductive and of Hermitian type. It follows that $N$ coincides with the nilradical (the maximal connected nilpotent normal Lie subgroup) of $G$. ing.

We have sketched the proof of the "only if part" of the follow-

Theorem 1. A connected unimodular (nonreductive) Lie group $G$ is a CS group if and only if it satisfies the following conditions.

(i) The nilradical $N$ of $G$ is isomorphic to a Heisenberg group $H_{n}$.

(ii) The reductive group $S=G / N$ is either of compact or of Hermitian type and its image under the natural homomorphism $\rho: S \rightarrow \operatorname{Out}(N)$ is contained in $\operatorname{Sp}(2 n, \mathbf{R})$.

(iii) If $S$ is of Hermitian type, there exists a $\rho$-equivariant holomorphic map from the Hermitian symmetric space $\mathscr{D}$ associated with $S$ to the Siegel space $\mathfrak{S}_{n}$.

That this theorem really classifies unimodular CS groups follows from the results of Satake (see [S2]) who classified $\rho$-equivariant holomorphic maps $\mathscr{D} \rightarrow \mathfrak{S}_{n}$ (this classification is closely related to the classification of Howe's reductive dual pairs in $\operatorname{Sp}(2 n, \mathbf{R})$ (cf. [Ho]).

\section{Classification of CS Representations}

Irreducible unitary representations of the groups which occur in Theorem 1 have been classified by Satake [S1]. Using his results and the results of the preceding sections (with Proposition 2 playing an essential role) we can complete the proof of Theorem 1 and also prove the following.

Theorem 2. Suppose $G$ has properties (i)-(iii) of Theorem 1. For any nonzero $\lambda \in \mathrm{X}(C)$, let $\left(\sigma_{\lambda}, \mathscr{F}_{\lambda}\right)$ be a projective representation of $G$ obtained by composing the (projective) metaplectic representation of (Aut $N)_{\lambda}$ (associated with $\left.\left(\beta_{\lambda}, \mathscr{F}_{\lambda}\right)\right)$ with $\tilde{\rho}$ and let $\alpha$ be its cocycle ( $\alpha$ does not depend on $\lambda$ and can be considered as a cocycle on $S=G / N)$. Let $\left(\pi_{1}, \mathscr{E}\right)$ be an irreducible projective 
unitary representation of $S$ with the following properties:

(i) its cocycle is $\alpha^{-1}$;

(ii) its kernel ker $\pi_{1}$ is contained in $N_{\mathscr{D}}(c f . \S 6)$;

(iii) the corresponding representation of $S / \mathrm{ker} \pi_{1}$ is a (projective) CS representation.

Then $(\pi, \mathscr{H})$, where $\mathscr{H}=\mathscr{E} \otimes \mathscr{F}_{\lambda}$ (Hilbert tensor product) and

$$
\pi(g)=\tilde{\pi}_{1}(g) \otimes \sigma_{\lambda}(g) \quad \text { for } g \in G,
$$

$\tilde{\pi}_{1}$ being the composition of $\pi_{1}$ and the projection $G \rightarrow S$, is a (linear) CS representation of $G$ and any CS representation of $G$ is of this form.

\section{REFERENCES}

[AM] R. Abraham and J. Marsden, Foundations of mechanics, 2nd ed., Benjamin/Cummings, Reading, MA, 1978.

[B1] F. A. Berezin, Quantization, Izv. Akad. Nauk SSSR Ser. Mat. 38 (1974), 1116-1175; English transl. in Math. USSR Izv. 38 (1974).

[B2] - Quantization in complex symmetric spaces, Izv. Akad. Nauk SSSR Ser. Mat. 39 (1975), 363-402; English transl. in Math. USSR Izv. 39 (1975).

[DN] J. Dorfmeister and K. Nakajima, The fundamental conjecture for homogeneous Kaehler manifolds, Acta Math. 161 (1988), 23-70.

[EHW] T. Enright, R. Howe and N. Wallach, $A$ classification of unitary highest weight modules, Representation Theory of Reductive Groups (P. Trombi, ed.), Progress in Math., vol. 40, Birkhäuser, Boston, 1983, pp. 97-143.

[Ha] J. I. Hano, On Kaehlerian homogeneous spaces of unimodular Lie groups, Amer. J. Math. 79 (1957), 885-900.

[Ho] R. Howe, $\theta$-series and invariant theory, Automorphic Forms, Representations and $L$-functions, Proc. Sympos. Pure Math., vol. 33, Amer. Math. Soc., Providence, RI, 1979, pp. 275-285.

[J] H. P. Jakobsen, Hermitian symmetric spaces and their unitary highest weight modules, J. Funct. Anal. 52 (1983), 385-412.

[K] S. Kobayashi, Irreducibility of certain unitary representations, J. Math. Soc. Japan 20 (1968), 638-642.

[KS] B. Kostant and S. Sternberg, Symplectic projective orbits, New Directions in Applied Mathematics, Springer-Verlag, Berlin and New York, 1982, pp. 81-84.

[L] W. Lisiecki, Kaehler coherent state orbits for representations of semisimple Lie groups, Ann. Inst. H. Poincaré Phys. Théor. 53 (1990), 245-258.

[O1] A. Odzijewicz, On reproducing kernels and quantization of states, Comm. Math. Phys. 114 (1988), 577-597.

[O2] - On the notion of mechanical system (to appear).

[P] A. M. Perelomov, Generalized coherent states and their applications, Springer-Verlag, Berlin and New York, 1986. 
[S1] I. Satake, Unitary representations of a semi-direct product of Lie groups on $\bar{\partial}$-cohomology spaces, Math. Ann. 190 (1971), 177-202.

[S2] _ Algebraic structures of symmetric domains, Princeton Univ. Press, Princeton, NJ, 1980.

[T] G. M. Tuynman, Studies in geometric quantization, Ph.D. thesis, Amsterdam, 1987.

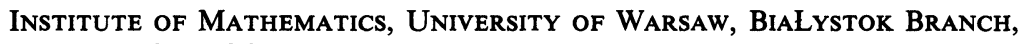
Akademicka 2, 15-267 Bialystok, Poland 
